\title{
Geoengineering: Whiter skies?
}

\author{
Ben Kravitz, ${ }^{1}$ Douglas G. MacMartin, ${ }^{2}$ and Ken Caldeira ${ }^{1}$ \\ Received 9 March 2012; revised 1 May 2012; accepted 2 May 2012; published 1 June 2012.
}

[1] One proposed side effect of geoengineering with stratospheric sulfate aerosols is sky whitening during the day and afterglows near sunset, as is seen after large volcanic eruptions. Sulfate aerosols in the stratosphere would increase diffuse light received at the surface, but with a non-uniform spectral distribution. We use a radiative transfer model to calculate spectral irradiance for idealized size distributions of sulfate aerosols. A 2\% reduction in total irradiance, approximately enough to offset anthropogenic warming for a doubling of $\mathrm{CO}_{2}$ concentrations, brightens the sky (increase in diffuse light) by 3 to 5 times, depending on the aerosol size distribution. The relative increase is less when optically thin cirrus clouds are included in our simulations. Particles with small radii have little influence on the shape of the spectra. Particles of radius $\sim 0.5 \mu \mathrm{m}$ preferentially increase diffuse irradiance in red wavelengths, whereas large particles $(\sim 0.9 \mu \mathrm{m})$ preferentially increase diffuse irradiance in blue wavelengths. Spectra show little change in dominant wavelength, indicating little change in sky hue, but all particle size distributions produce an increase in white light relative to clear sky conditions. Diffuse sky spectra in our simulations of geoengineering with stratospheric aerosols are similar to those of average conditions in urban areas today. Citation: Kravitz, B., D. G. MacMartin, and K. Caldeira (2012), Geoengineering: Whiter skies?, Geophys. Res. Lett., 39, L11801, doi:10.1029/2012GL051652.

\section{Introduction}

[2] Robock [2008, 2011] argued that geoengineering with sulfate aerosols would cause whitening of the sky, as was seen after the eruption of Mount Pinatubo [Robock, 2000]. Geoengineering with stratospheric sulfate aerosols to offset anthropogenic warming [Crutzen, 2006] would result in direct irradiance being decreased by much more than total irradiance, which is compensated by an increase in diffuse irradiance. When the ratio of particle size to scattered wavelength is small, such as for air molecules scattering visible wavelengths (380-780 nm), scattering is in the Rayleigh regime, in which short wavelengths (blues) are scattered more efficiently than long wavelengths (reds), giving the sky its blue hue. When particles are similar in size to the scattered wavelength, as is the case for stratospheric sulfate aerosols from large volcanic eruptions, scattering is more uniform

\footnotetext{
${ }^{1}$ Department of Global Ecology, Carnegie Institution for Science, Stanford, California, USA.

${ }^{2}$ Department of Control and Dynamical Systems, California Institute of Technology, Pasadena, California, USA.

Corresponding author: B. Kravitz, Department of Global Ecology, Carnegie Institution for Science, 260 Panama St., Stanford, CA 94305, USA. (bkravitz@carnegie.stanford.edu)

Copyright 2012 by the American Geophysical Union. 0094-8276/12/2012GL051652
}

across the visible spectrum. Thus, in the presence of an aerosol layer, diffuse irradiance at longer wavelengths (reds) increases more than the increase in shorter wavelengths (blues). As scattering increases, the portion of diffuse light also increases, resulting in a brighter sky as seen from the ground, because sulfate aerosols preferentially forward scatter more radiation than they back scatter.

[3] In this paper, we use the term brightness of sky to refer to diffuse irradiance integrated over the visible band from an observer looking upward, and we are specifically excluding direct irradiance of the sun in reporting this quantity. We refer to white light as having a uniform irradiance spectrum, so a whiter sky would have a higher portion of irradiance in longer wavelengths than a blue sky. All references to solar irradiance reduction are in irradiance integrated over the visible band as measured at the surface.

\section{Simulations}

[4] To determine the degree to which the sky will whiten and brighten as a result of idealized distributions of stratospheric sulfate aerosols, we use the radiative transfer model libRadtran [Mayer and Kylling, 2005] (see Text S1 in the auxiliary material) to simulate spectral irradiance. ${ }^{1}$ Because the scattering properties of sulfate aerosols are strongly dependent upon the size distribution, we compare the results for lognormal distributions with different median radii and standard deviations, the latter of which is a measure of distribution width. We investigate atmospheric loadings of aerosols that correspond to up to a $2 \%$ reduction in irradiance received at the surface, which is roughly the amount needed to offset the warming from a doubling of $\mathrm{CO}_{2}$ concentrations from the preindustrial era [Govindasamy and Caldeira, 2000].

\section{Spectral Irradiance and Mie Calculations}

[5] Figure 1 shows perturbations to the visible light spectrum from our simulations of stratospheric sulfate aerosols (unimodal lognormal, median radius $r_{g}=0.5 \mu \mathrm{m}, \sigma=0.1$ ) with an aerosol optical depth that reduces total integrated visible band surface irradiance by $2 \%$. Relative to a clear sky case (no clouds or aerosols), a $2 \%$ reduction in total irradiance with this aerosol size distribution reduces daytime direct irradiance by $22 \%$ and increases diffuse irradiance by over four times. The increase in diffuse irradiance is more prominent in red wavelengths than blue wavelengths.

[6] For our daytime simulations, we use a solar zenith angle of $0^{\circ}$. We examined the impacts of changing solar zenith angle on the aerosol effects on irradiance spectra. Except for large solar zenith angles (i.e., near sunrise or sunset), increasing the angle reduces irradiance by a factor

\footnotetext{
${ }^{1}$ Auxiliary materials are available in the HTML. doi:10.1029/ 2012GL051652.
} 

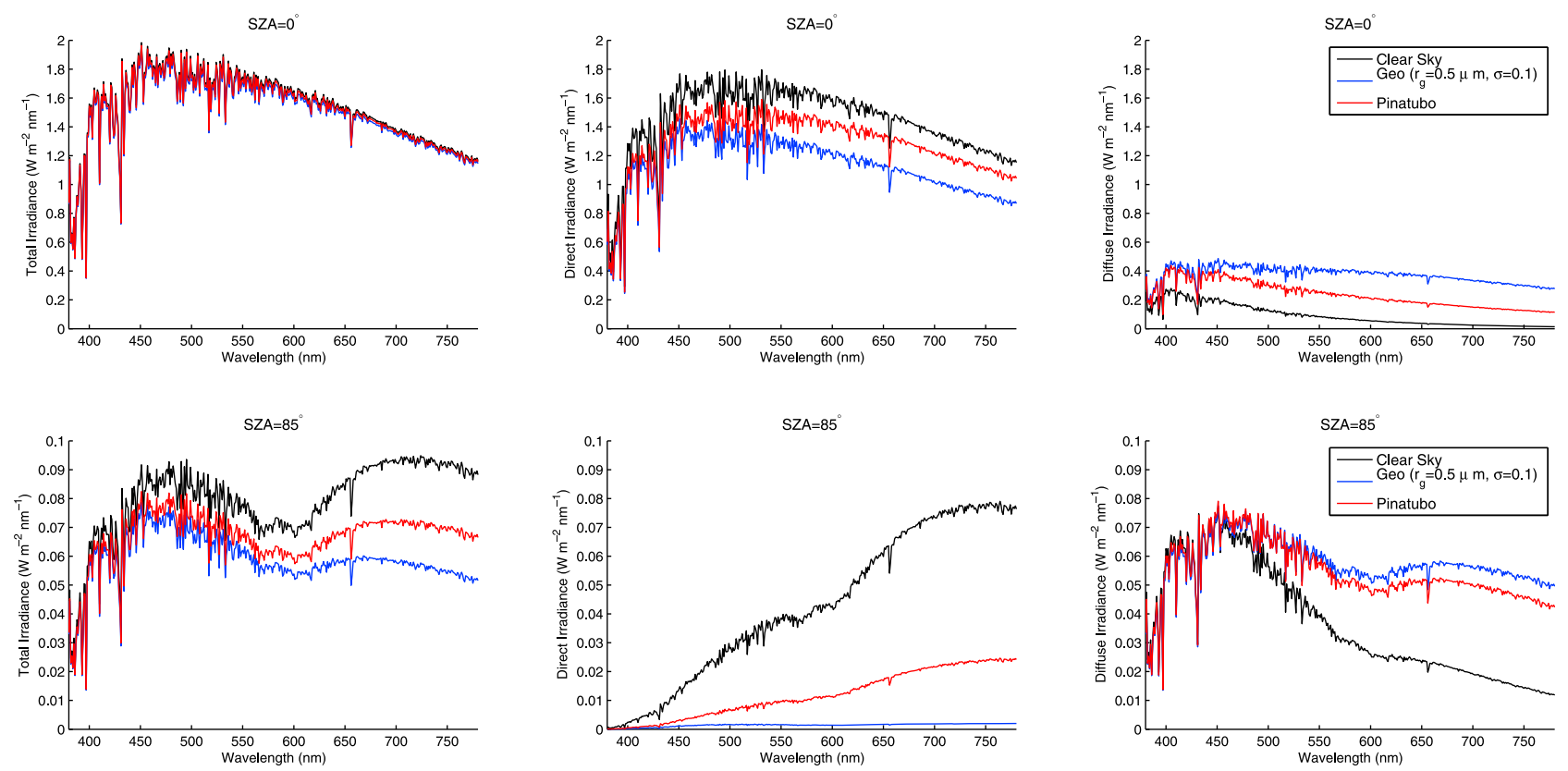

Figure 1. Spectral irradiances for clear sky, idealized geoengineering, and Pinatubo simulations. (top) A solar zenith angle of $0^{\circ}$, which represents daytime conditions, and (bottom) a solar zenith angle of $85^{\circ}$, which represents conditions at sunset (with different $y$-axis). (left) Total irradiance received at the surface, (middle) direct irradiance, and (right) diffuse irradiance. Sulfate aerosol results are shown for a unimodal lognormal distribution with $r_{g}=0.5 \mu \mathrm{m}, \sigma=0.1$, and an aerosol optical depth corresponding to a $2 \%$ reduction in integrated visible surface irradiance, which is approximately the amount needed to offset the forcing from a doubling of $\mathrm{CO}_{2}$ concentrations from preindustrial.

that is nearly constant at all wavelengths, so daytime sky hue changes from stratospheric aerosols are relatively insensitive to solar zenith angle (not shown). At large solar zenith angles, the sun appears redder than in daytime, as the amount of atmosphere through which light must travel to reach an observer increases. This increases the amount of Rayleigh scattering by air molecules, which preferentially scatters shorter wavelengths, and consequently longer wavelengths (reds and oranges) reach the observer as a higher portion of light [Corfidi, 1996]. Therefore, direct solar radiation at sunset appears to be redder, whereas the diffuse sky retains its blue hue (Figure 1, bottom). However, in the presence of a stratospheric aerosol layer, solar radiation is scattered by the aerosol layer, resulting in an afterglow that is characteristic of volcanic sunsets [Corfidi, 1996]. Indeed, our simulations for the eruption of Pinatubo (described in Section 3 of Text S1) show an increase in diffuse light by approximately a factor of two at long wavelengths; direct irradiance decreases by up to $75 \%$ at long wavelengths. Similar to the eruption of Pinatubo, sulfate aerosols under the specifications we simulated $\left(\mathrm{r}_{g}=0.5 \mu \mathrm{m}, \sigma=0.1\right)$ would cause sunsets similar in appearance to volcanic sunsets.

[7] Changes in irradiance are dependent upon the size distribution of the particles, both in terms of median radius and distribution width. Figure 2 shows spectral irradiance and asymmetry parameters for a range of median radii and distribution standard deviations. The asymmetry parameter $g$ indicates the average direction of light scattering. Pure backscattering corresponds to $g=-1$, pure forward scattering to $g=1$, and isotropic scattering to $g=0$. Note that $g$ varies with wavelength in ways that depend on the particle size distribution. All cases show at least a factor of 2 increase in diffuse irradiance over the clear sky case, indicating a sky brightening.

[8] The distribution with median radius $r_{g}=0.1 \mu \mathrm{m}$ shows an asymmetry parameter that is almost uniform in wavelength, meaning the same approximate percentage of diffuse light will be forward scattered at all wavelengths. Because the solar spectrum has more irradiance in shorter wavelengths than longer ones, the diffuse spectrum resulting from stratospheric sulfate aerosols with this particular size distribution has a larger increase in irradiance in blue wavelengths than red wavelengths. The results for $r_{g}=0.1$ and $0.3 \mu \mathrm{m}$ are similar. For $r_{g}=0.5 \mu \mathrm{m}$, the asymmetry parameter favors forward scattering of longer wavelengths, resulting in a flattening of the diffuse spectrum. This whitens the sky, where white light is defined as having uniform irradiance for all visible wavelengths. $r_{g}=0.7 \mu \mathrm{m}$ has an asymmetry parameter that favors forward scattering of both short and long visible wavelengths, and $r_{g}=0.9 \mu \mathrm{m}$ favors forward scattering of short wavelengths. The latter results in the smallest change in the overall shape of the diffuse spectrum.

[9] The asymmetry parameter depends more strongly on wavelength for narrower size distributions. For a distribution median radius of $r_{g}=0.5 \mu \mathrm{m}$, as the distribution width increases, the asymmetry parameter becomes more uniform, slightly favoring forward scattering of shorter wavelengths. The asymmetry parameter also increases (more forward scattering) for all wavelengths when $\sigma$ changes from 0.5 to 1.0 , which skews diffuse irradiance toward shorter wavelengths.

[10] Table 1 shows increases in diffuse sky brightness (diffuse irradiance integrated over the visible band) for all simulations we performed. Larger reductions in solar irradiance are achieved by larger optical depths (Table 2 in 

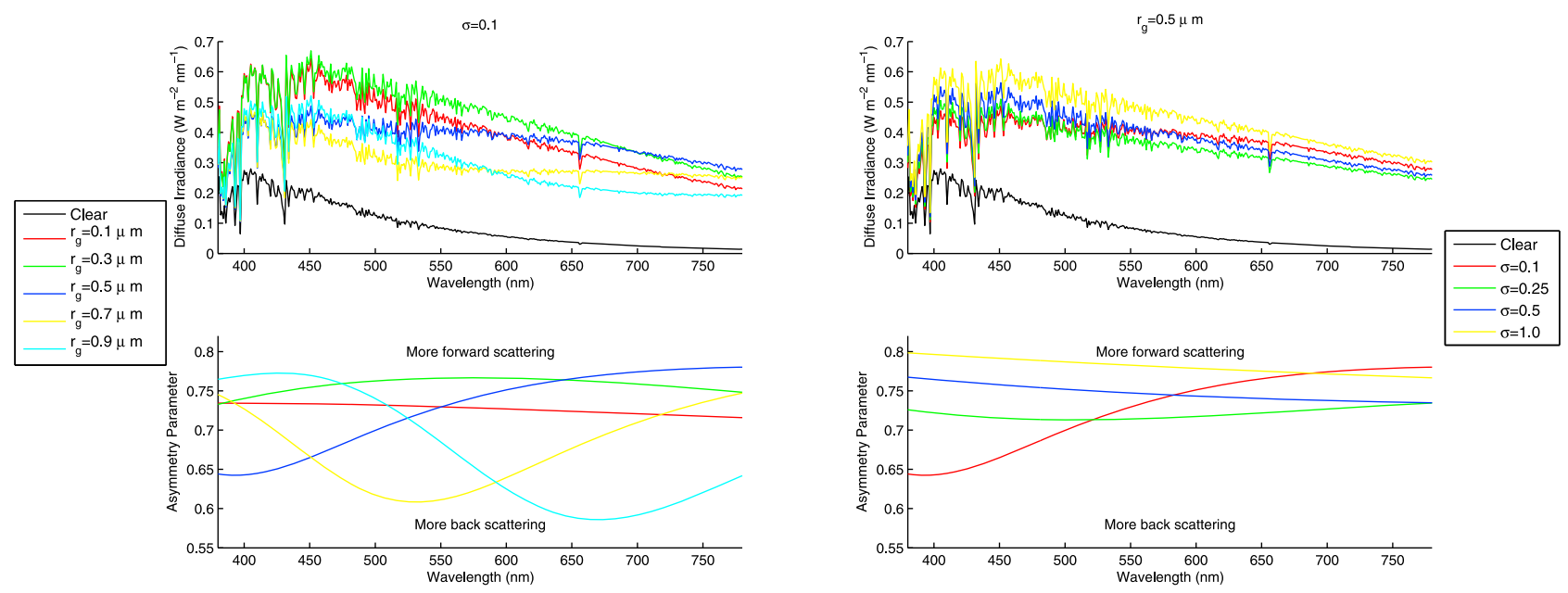

Figure 2. The dependence of diffuse irradiance spectra and asymmetry parameter on median radius $\left(r_{g}\right)$ and standard deviation $(\sigma)$ of the unimodal lognormal distribution for stratospheric sulfate aerosols. (left) Radius is varied, but $\sigma$ is fixed at 0.1, which is a narrow distribution and serves to isolate the effect of changing radius. (right) $r_{g}$ is fixed at $0.5 \mu \mathrm{m}$ and varied $\sigma$. (top) The clear sky case (no aerosols) is shown for comparison.

Text S1), so large reductions have more sky brightening. Increasing $\sigma$ from 0.1 to 0.25 decreases brightness for small distribution median radii, and increasing $\sigma$ from 0.25 to 0.5 or 1.0 increases brightness. This can be explained by integrating under the irradiance curves in Figure 2 (top right). For large median radii, the increase in brightness is monotonic with $\sigma$.

[11] The dependence of brightness on the median radius of the distribution strongly depends upon the distribution width (Table 1). For narrow distributions $(\sigma=0.1)$, the radius that gives the largest increase in brightness is $r_{g}=0.3 \mu \mathrm{m}$, and the smallest increase is for $r_{g}=0.8 \mu \mathrm{m}$. For $\sigma \geq 0.25$, the largest brightness increase is for the smallest radius of $r_{g}=$ $0.1 \mu \mathrm{m}$, and brightness increases diminish almost monotonically with increasing radius.

[12] We also performed simulations of thin cirrus clouds to determine the effects of these clouds on our simulations (see Section 4 of Text S1). A cloud fraction of 20\% [Mace et al., 2001] diminishes brightness increases by approximately $40 \%$ for all size distributions (Table 3 in Text S1). Effects of the geoengineering case $r_{g}=0.5 \mu \mathrm{m}, \sigma=0.1$ show nearly negligible changes in total irradiance, up to a $5 \%$ decrease in direct irradiance, and over a $20 \%$ increase in diffuse irradiance (Figure 1 in Text S1). The enhancement of diffuse light by cirrus clouds is by an approximately constant factor across all wavelengths, whereas stratospheric sulfate aerosols preferentially enhance longer wavelengths over shorter ones.

\section{Perceived Color}

[13] Determining whether the sky would be whiter under geoengineering is not straightforward. The main difficulty in determining this effect is that color is not an intrinsic property of light; rather, it is perceived and interpreted. Therefore, the relevance of showing color swatches of sky color in red-green-blue color space (or another equivalent) is not immediately apparent, as it is difficult to interpret these color swatches out of their normal perceptual context. Sky color temperature varies with sunlight and meteorological conditions, and many different color matching algorithms have been proposed, all of which can radically affect color perception. (See Section 7 of Text S1 for a discussion and examples.) Furthermore, there is no guarantee the colors will be displayed with fidelity, as color appearance depends on the means of viewing, e.g., the computer monitor and printer.

[14] Despite these difficulties, one can obtain some measure of sky color change. A metameric match is the monochromatic wavelength which will elicit the same response in the retinas as a specified irradiance spectrum. Using an average profile of cone sensitivities [Stockman and Sharpe, 2000] and matching functions [Smith, 2005] (see Section 6 of Text S1), we determined the metameric matches to spectra produced by our simulations. Additionally, the perceived sky can be approximated as a mixture of this monochromatic light with white light (light with a uniform irradiance spectrum).

Table 1. Increases in Visible Band Integrated Diffuse Irradiance as a Measure of Diffuse Sky Brightness ${ }^{\text {a }}$

\begin{tabular}{clccccccccc}
\hline & & \multicolumn{10}{c}{$\left[r_{g}(\mu \mathrm{m})\right]$} \\
\cline { 2 - 10 }$\Delta I(\%)$ & $\sigma$ & 0.1 & 0.2 & 0.3 & 0.4 & 0.5 & 0.6 & 0.7 & 0.8 & 0.9 \\
\hline 0.5 & 0.1 & 1.95 & 2.07 & 2.10 & 2.00 & 1.86 & 1.72 & 1.62 & 1.60 & 1.62 \\
& 0.25 & 1.88 & 1.87 & 1.85 & 1.82 & 1.78 & 1.74 & 1.71 & 1.71 & 1.73 \\
& 0.5 & 1.97 & 1.95 & 1.92 & 1.90 & 1.89 & 1.87 & 1.87 & 1.87 & 1.88 \\
& 1.0 & 2.10 & 2.11 & 2.10 & 2.10 & 2.09 & 2.09 & 2.09 & 2.09 & 2.09 \\
1.0 & 0.1 & 2.83 & 3.06 & 3.10 & 2.93 & 2.66 & 2.39 & 2.22 & 2.17 & 2.22 \\
& 0.25 & 2.71 & 2.69 & 2.66 & 2.60 & 2.52 & 2.44 & 2.39 & 2.38 & 2.41 \\
& 0.5 & 2.88 & 2.83 & 2.77 & 2.75 & 2.72 & 2.69 & 2.68 & 2.68 & 2.70 \\
& 1.0 & 3.12 & 3.13 & 3.12 & 3.11 & 3.10 & 3.09 & 3.09 & 3.09 & 3.09 \\
1.5 & 0.1 & 3.65 & 3.96 & 4.03 & 3.80 & 3.41 & 3.03 & 2.78 & 2.71 & 2.78 \\
& 0.25 & 3.48 & 3.45 & 3.41 & 3.32 & 3.21 & 3.10 & 3.03 & 3.01 & 3.06 \\
& 0.5 & 3.71 & 3.65 & 3.59 & 3.54 & 3.49 & 3.46 & 3.44 & 3.45 & 3.47 \\
& 1.0 & 4.05 & 4.07 & 4.06 & 4.04 & 4.03 & 4.01 & 4.01 & 4.01 & 4.02 \\
2.0 & 0.1 & 4.41 & 4.79 & 4.88 & 4.59 & 4.11 & 3.63 & 3.32 & 3.22 & 3.32 \\
& 0.25 & 4.20 & 4.16 & 4.11 & 4.00 & 3.86 & 3.72 & 3.63 & 3.61 & 3.68 \\
& 0.5 & 4.49 & 4.42 & 4.34 & 4.27 & 4.21 & 4.17 & 4.15 & 4.16 & 4.19 \\
& 1.0 & 4.91 & 4.93 & 4.92 & 4.90 & 4.88 & 4.87 & 4.86 & 4.86 & 4.87 \\
\hline
\end{tabular}

${ }^{\mathrm{a}} \mathrm{Leftmost}$ column indicates the percent decrease in total integrated visible band irradiance. All values given are ratios of integrated visible band irradiance (stratospheric sulfate aerosols divided by clear sky) and are rounded to two decimal places. In the clear sky simulations, diffuse irradiance comprises approximately $6 \%$ of total irradiance. 

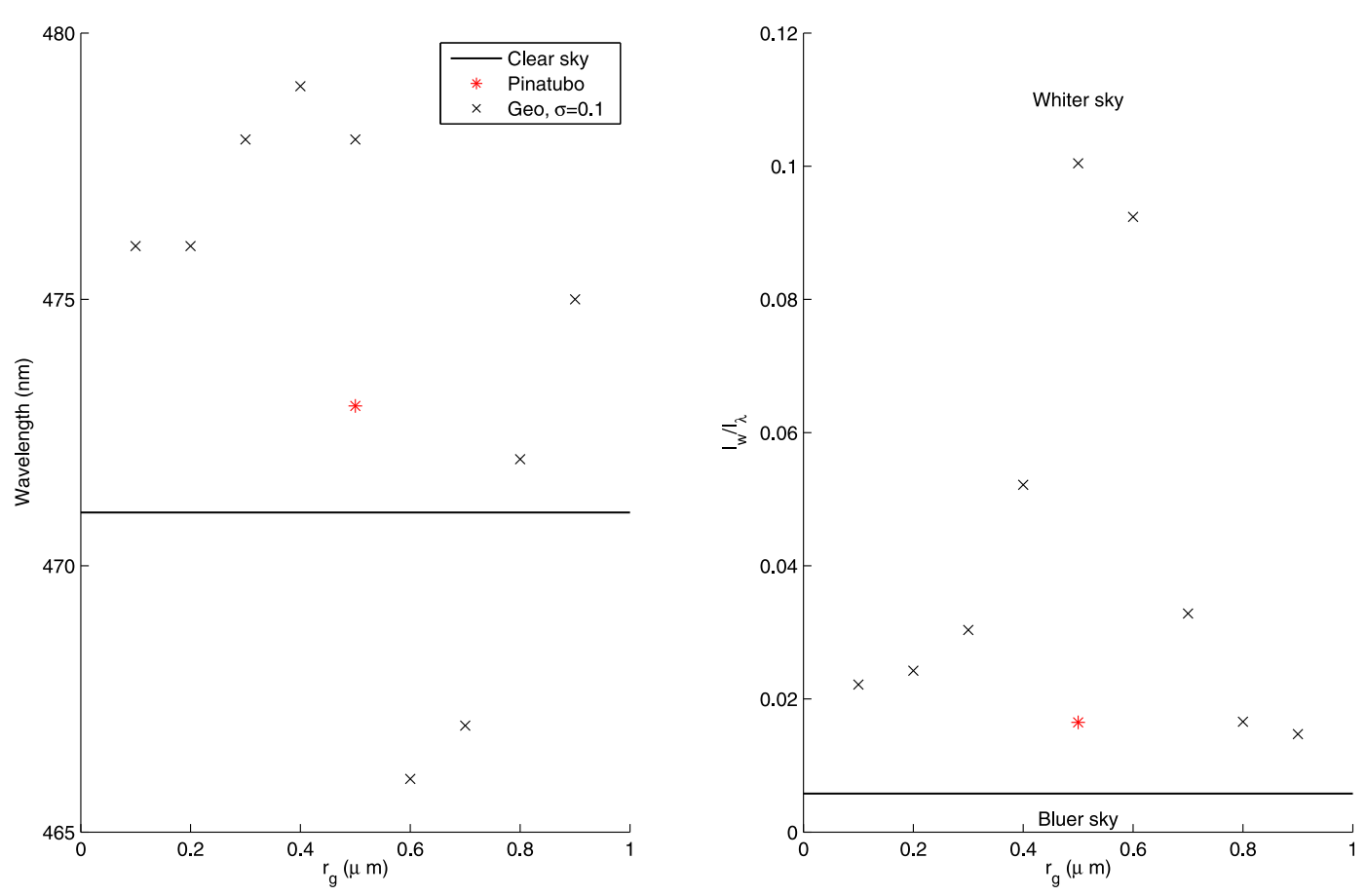

Figure 3. Metameric matches of irradiance spectra to perceived dominant wavelength. Left panel is dependence upon radius of perceived diffuse sky wavelength due to aerosol injection. Right panel shows the ratio of white (uniform spectrum) to monochromatic light, with higher values indicating a perceived whiter sky. All stratospheric sulfate aerosol values shown are for a $2 \%$ reduction in visible integrated total surface irradiance, with $\sigma$ fixed at 0.1 to highlight radius dependence. The clear sky case (no aerosols) is shown as black horizontal lines. Red asterisks show simulations for 9 months after the 1991 eruption of Mount Pinatubo, which has a wider aerosol size distribution.

[15] Figure 3 illustrates these two concepts for our simulations, assuming a $2 \%$ reduction in visible integrated solar irradiance and a narrow size distribution to highlight the effects of radius. The black lines in Figure 3 show the case for a clear sky (no aerosols). Small radii show the largest wavelength departure, reaching a maximum difference of $8 \mathrm{~nm} . r_{g}=0.8 \mu \mathrm{m}$ shows the smallest departure by only $1 \mathrm{~nm}$. All of the wavelengths in Figure 3 fall distinctly within the blue range [Boynton et al., 1964], so none of these departures is indicative of a radical change in sky color. However, all simulations pictured in Figure 3 show a whitening of the sky. The white to monochromatic light ratio increases by over a factor of 2 for all cases, and by a full order of magnitude for $r_{g}=0.5$ and $0.6 \mu \mathrm{m}$. This figure shows that aerosols in the middle of the range we considered would likely cause the largest changes in perceived color. For reference, we have also included a calculation for the 1991 eruption of Mount Pinatubo (red asterisks, see Section 3 of Text S1 for specifications of the simulation). Nearly all of our geoengineering simulations that reduce total irradiance by $2 \%$ show more sky whitening than was seen in March after the eruption of Pinatubo.

\section{Comparisons: Urban Pollution}

[16] To provide context for our results, we compare the spectra from our stratospheric sulfate aerosol geoengineering simulations with spectra characteristic of a range of urban air pollution conditions. Since pollution in urban areas varies on a daily basis, depending upon meteorological conditions, as well as the particular urban area, we compared our results to an average of aerosol measurements (see Table 1 in Text S1) taken in three urban areas (Greenbelt, MD; Paris, France; and Mexico City, Mexico), including a low aerosol case (relatively clear day), a high aerosol case (relatively polluted day), and an average/typical case [Dubovik et al., 2002] (see Section 5 of Text S1). Stratospheric aerosols with a size distribution specified by $r_{g}=0.5 \mu \mathrm{m}$ and $\sigma=1.0$ and with a reduction of visible integrated solar irradiance by $\sim 1.5 \%$ have a similar spectrum to an average pollution day in the urban areas considered (Figure 4). A day with heavy pollution would correspond to a level of stratospheric sulfate aerosols that would produce a solar irradiance reduction far exceeding $2 \%$. For reference, we have also included a simulation of the 1991 eruption of Mount Pinatubo (specifications in Section 3 of Text S1).

[17] For stratospheric sulfate aerosol distributions with $r_{g}=$ $0.5 \mu \mathrm{m}$ and $\sigma=1.0$, an optical depth of 0.15 results in a solar irradiance reduction of $1 \%$, and reduction in irradiance scales linearly with optical depth (Table 2 in Text S1). Therefore, assuming the average urban pollution case has an optical depth of $\tau_{550}=0.21$, an additional solar reduction of $1 \%$ from stratospheric aerosols would, on average, cause the sky over formerly pristine areas to look similar to the sky over urban areas. The variation of optical depth in urban areas that already occurs is much larger than the added optical depth from our simulations.

\section{Conclusions}

[18] According to our simulations, stratospheric geoengineering with sulfate aerosols at the levels considered here likely would not cause profound changes in sky hue but would whiten the sky, potentially exceeding the amount of 

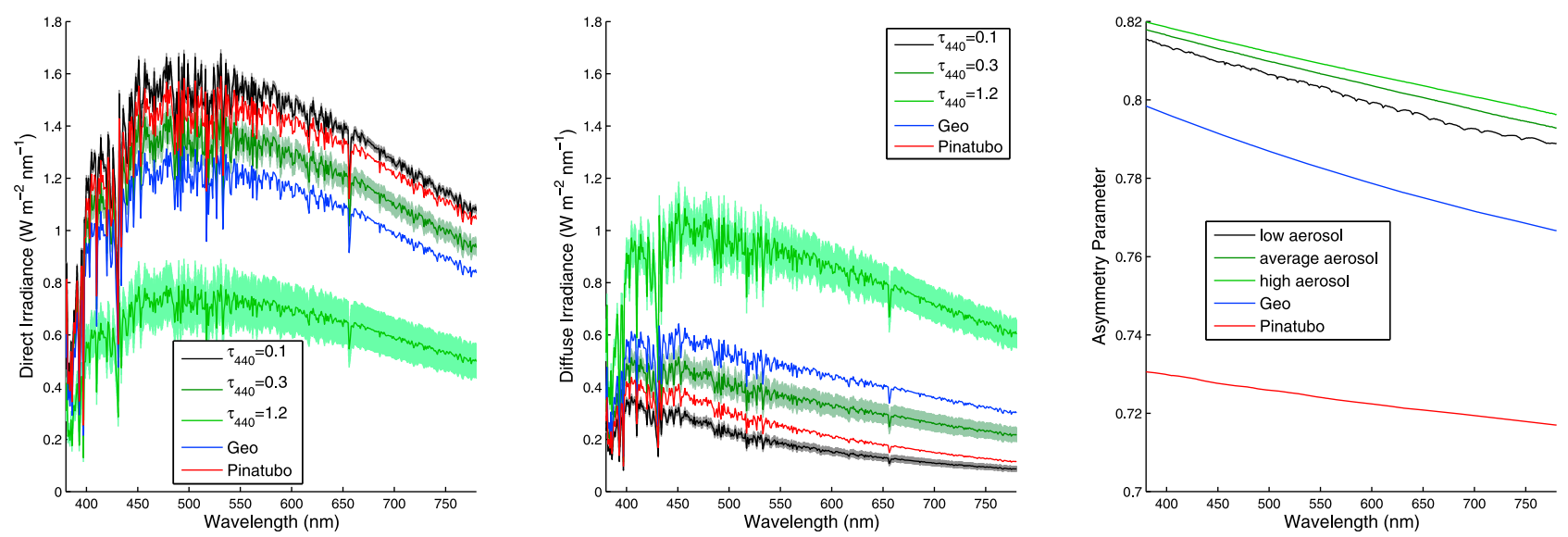

Figure 4. Direct and diffuse irradiance spectra and asymmetry parameter for different cases of urban aerosols (assumed to be sulfate aerosols, as discussed in Section 5 of Text S1), in which optical depth and Angstrom exponent are varied. All values used in calculations are taken as averages of urban aerosol values [Dubovik et al., 2002]. Shaded areas indicate a range of observed Angstrom exponents for the indicated optical depth, and solid colored lines indicate average conditions. Blue lines are an idealized sulfate aerosol distribution case with $r_{g}=0.5 \mu \mathrm{m}$ and $\sigma=1.0$, with an aerosol optical depth resulting in a $2 \%$ reduction in total surface irradiance. Red lines correspond to a simulation of the 1991 eruption of Mount Pinatubo, with conditions from March 1992.

whitening observed after the 1991 eruption of Mount Pinatubo. These levels of geoengineering would also cause sunsets similar to those seen after large volcanic eruptions.

[19] The results of this study have multiple implications, although some are not easily quantified. One of the motivations for Robock [2008] is the concern over potential psychological implications. We have provided the physics-based predictions of sky changes from stratospheric sulfate geoengineering, and our results can provide a basis for psychological research. Additionally, public perception of the effects discussed in this paper could be investigated, as the whitening of skies could contribute to opposition to geoengineering.

[20] One of the more quantifiable impacts is a potential enhancement of the land carbon sink, as was seen after the 1991 eruption of Mount Pinatubo [Mercado et al., 2009]. Diffuse light more easily penetrates canopies, and it can also more easily strike more leaf surfaces, which can increase plant photosynthetic activity. However, the degree to which this effect could occur depends on the size distribution of the sulfate aerosols, as our results show.

[21] Additionally, solar power generating capacity could be affected by geoengineering [Murphy, 2009]. Generation of solar power by concentrating solar thermal plants is largely proportional to the amount of direct sunlight received. Therefore, the reductions in direct irradiance that our results show could reduce the effectiveness of this particular method of obtaining low-carbon-emission energy, which could lead to increased use of other energy sources, including fossil fuel combustion.

[22] Although we do not discuss photochemical impacts, Tilmes et al. [2008] have shown that sulfate aerosol geoengineering has the potential to decrease stratospheric ozone concentrations. Possible impacts of sulfate geoengineering on chemistry warrant further study.

[23] Different means of generating the aerosol particles would produce different aerosol size distributions, affecting development of engineering approaches to geoengineering. If the aerosols are formed from photochemical conversion of $\mathrm{SO}_{2}$ into sulfate aerosols, the expected particle size distribution width is large [Heckendorn et al., 2009], meaning the median particle radius has diminished importance in determining the amount of sky whitening, but sky brightening will be larger than for narrower distributions. However, if a monodisperse size distribution of aerosols is created, which could result from direct condensation of gaseous sulfuric acid [Pierce et al., 2010], median radius becomes important in determining sky whiteness. Our results also have implications for geoengineering with engineered particles, in that the sky color and brightness will depend very strongly on the asymmetry parameter of the particle.

[24] To achieve a minimal amount of sky whitening under geoengineering with stratospheric sulfate aerosols, there are three options: (1) choose a larger particle size so the particles preferentially forward scatter more in blue wavelengths than red wavelengths (the shape of $g$ will be similar to that of $\bar{r}=0.9 \mu \mathrm{m}$ in Figure 2); (2) Use particles that have less forward scattering in all wavelengths (smaller value of $g$ ); or (3) Limit the amount of sulfate geoengineering or avoid such geoengineering entirely. The first option is problematic, as large aerosols have a higher fall speed than smaller aerosols, meaning a higher rate of injection is needed for these large aerosols than for smaller ones to achieve the same amount of atmospheric mass loading. The second is problematic, as can be seen from Figure 2, because all size distributions of sulfate aerosols considered scatter prominently in the forward direction. Thus, an obvious path to avoiding sky whitening is to greatly limit or avoid entirely the intentional introduction of sulfate particles into the stratosphere.

[25] Acknowledgments. We thank Alan Robock for suggesting this topic and for helpful comments throughout the process of drafting this paper, as well as the reviewers for their comments.

[26] The Editor thanks the anonymous reviewers for assisting with the evaluation of this paper.

\section{References}

Boynton, R. M., W. Schafer, and M. E. Neun (1964), Hue-wavelength relation measured by color-naming method for three retinal locations, Science, 146, 666-668. 
Corfidi, S. F. (1996), The colors of twilight, Weatherwise, 49(3), 14-19, doi:10.1080/00431672.1996.9925403.

Crutzen, P. J. (2006), Albedo enhancement by stratospheric sulfur injections: A contribution to resolve a policy dilemma? Climatic Change, 77, 211-219, doi:10.1007/s10584-006-9101-y.

Dubovik, O., et al. (2002), Variability of absorption and optical properties of key aerosol types observed in worldwide locations, J. Atmos. Sci., 59, 590-608.

Govindasamy, B., and K. Caldeira (2000), Geoengineering Earth's radiation balance to mitigate $\mathrm{CO}_{2}$-induced climate change, Geophys. Res. Lett., 27, 2141-2144, doi:10.1029/1999GL006086.

Heckendorn, P., et al. (2009), The impact of geoengineering aerosols on stratospheric temperature and ozone, Environ. Res. Lett., 4, 045108, doi:10.1088/1748-9326/4/4/045108.

Mace, G. G., E. E. Clothiaux, and T. P. Ackerman (2001), The composite characteristics of cirrus clouds: Bulk properties revealed by one year of continuous cloud data, J. Clim., 14, 2185-2203.

Mayer, B., and A. Kylling (2005), Technical note: The libRadtran software package for radiative transfer calculations-Description and examples of use, Atmos. Chem. Phys., 5, 1855-1857, doi:10.5194/acp-5-1855-2005.

Mercado, L. M., N. Bellouin, S. Sitch, O. Boucher, C. Huntingford, M. Wild, and P. M. Cox (2009), Impact of changes in diffuse radiation on the global land carbon sink, Nature, 458, 1014-1017, doi:10.1038/ nature07949.
Murphy, D. M. (2009), Effect of stratospheric aerosols on direct sunlight and implications for concentrating solar power, Environ. Sci. Technol, 48(8), 2784-2786, doi:10.1021/es802206b.

Pierce, J. R., D. K. Weisenstein, P. Heckendorn, T. Peter, and D. W. Keith (2010), Efficient formation of stratospheric aerosol for climate engineering by emission of condensible vapor from aircraft, Geophys. Res. Lett., 37, L18805, doi:10.1029/2010GL043975.

Robock, A. (2000), Volcanic eruptions and climate, Rev. Geophys., 38, 191-219, doi:10.1029/1998RG000054.

Robock, A. (2008), 20 reasons why geoengineering may be a bad idea, Bull. At. Sci., 64, 14-18, doi:10.2968/064002006.

Robock, A. (2011), Bubble, bubble, toil and trouble: An editorial comment, Clim. Change, 105, 383-385, doi:10.1007/s10584-010-0017-1.

Smith, G. S. (2005), Human color vision and the unsaturated blue color of the daytime sky, Am. J. Phys., 73, 590-597, doi:10.1119/1.1858479.

Stockman, A., and L. T. Sharpe (2000), The spectral sensitivites of the middle and long-wavelength-sensitive cones derived from measurements in observers of known genotype, Vision Res., 40, 1711-1737.

Tilmes, S., R. Müller, and R. Salawitch (2008), The sensitivity of polar ozone depletion to proposed geoengineering schemes, Science, 320 , 1201-1204, doi:10.1126/science.1153966. 